\title{
International expert consensus on the management of bleeding during VATS lung surgery
}

\author{
Lunxu Liu ${ }^{1}$, Jiandong $\mathrm{Mei}^{1}$, Jie $\mathrm{He}^{2}$, Todd L. Demmy ${ }^{3}$, Shugeng Gao ${ }^{2}$, Shanqing $\mathrm{Li}^{4}$, Jianxing $\mathrm{He}^{5,6}$, \\ Yang Liu ${ }^{7}$, Yunchao Huang ${ }^{8}$, Shidong $\mathrm{Xu}^{9}$, Jian $\mathrm{Hu}^{10}$, Liang Chen ${ }^{11}$, Yuming Zhu ${ }^{12}$, Qingquan Luo ${ }^{13}$, \\ Weimin Mao ${ }^{14}$, Qunyou Tan ${ }^{15}$, Chun Chen ${ }^{16}$, Xiaofei Li ${ }^{17}$, Zhu Zhang ${ }^{18}$, Gening Jiang ${ }^{12}$, Lin Xu ${ }^{19}$, \\ Lanjun Zhang ${ }^{20}$, Jianhua Fu ${ }^{20}$, Hui Li ${ }^{21}$, Qun Wang ${ }^{22}$, Deruo Liu ${ }^{23}$, Lijie Tan ${ }^{24}$, Qinghua Zhou ${ }^{24}$, \\ Xiangning $\mathrm{Fu}^{25}$, Zhongmin Jiang ${ }^{26}$, Haiquan Chen ${ }^{27}$, Wentao Fang ${ }^{28}$, Xun Zhang ${ }^{29}$, Yin $\mathrm{Li}^{2}$, Ti Tong ${ }^{30}$, \\ Zhentao Yu ${ }^{31}$, Yongyu Liu ${ }^{32}$, Xiuyi Zhi ${ }^{33}$, Tiansheng Yan $^{34}$, Xingyi Zhang ${ }^{35}$, Qiang Pu ${ }^{1}$, Guowei Che ${ }^{1}$, \\ Yidan Lin ${ }^{1}$, Lin $\mathrm{Ma}^{1}$, Raul Embun ${ }^{36}$, Javier Aragón ${ }^{37}$, Serdar Evman ${ }^{38}$, Gregor J. Kocher ${ }^{39}$, Luca Bertolaccini ${ }^{40}$, \\ Alessandro Brunelli ${ }^{41}$, Diego Gonzalez-Rivas ${ }^{42}$, Joel Dunning ${ }^{43}$, Hui-Ping Liu ${ }^{44}$, Scott J. Swanson ${ }^{45}$, \\ Ryabov Andrey Borisovich ${ }^{46}$, Inderpal S. Sarkaria ${ }^{47}$, Alan Dart Loon Sihoe ${ }^{48}$, Takeshi Nagayasu ${ }^{49}$, \\ Takuro Miyazaki ${ }^{49}$, Masayuki Chida ${ }^{50}$, Tadasu Kohno ${ }^{51}$, Agasthian Thirugnanam ${ }^{52}$, Harmic J. Soukiasian ${ }^{53}$, \\ Mark W. Onaitis ${ }^{54}$, Chia-Chuan Liu ${ }^{55}$; on behalf of International Interest Group on Bleeding during \\ VATS Lung Surgery
}

${ }^{1}$ Department of Thoracic Surgery, West China Hospital, Sichuan University, Chengdu 610041, China; ${ }^{2}$ Department of Thoracic Surgery, National Cancer Center/National Clinical Research Center for Cancer/Cancer Hospital, Chinese Academy of Medical Sciences and Peking Union Medical College, Beijing 100021, China; ${ }^{3}$ Department of Thoracic Surgery, Roswell Park Cancer Institute, Buffalo, NY, USA; ${ }^{4}$ Department of Thoracic Surgery, Peking Union Medical College Hospital, Chinese Academy of Medicine, Beijing 100032, China; ${ }^{5}$ Department of Thoracic Surgery, The First Affiliated Hospital of Guangzhou Medical University, Guangzhou 510120, China; ${ }^{6}$ Guangzhou Institute of Respiratory Disease \& China State Key Laboratory of Respiratory Disease \& National Clinical Research Center for Respiratory Disease, Guangzhou 510120, China; ${ }^{7}$ Department of Thoracic Surgery, Chinese PLA General Hospital, Beijing 100853, China; ${ }^{8}$ Department of Thoracic and Cardiovascular Surgery, The Third Affiliated Hospital of Kunming Medical University (Yunnan Cancer Hospital), Kunming 650106, China; ${ }^{9}$ Department of Thoracic Surgery, Harbin Medical University Cancer Hospital, Harbin 150086, China; ${ }^{10}$ Department of Thoracic Surgery, First Hospital Affiliated to Medical College of Zhejiang University, Hangzhou 310003, China; ${ }^{11}$ Department of Thoracic Surgery, The First Affiliated Hospital of Nanjing Medical University, Nanjing 210029, China; ${ }^{12}$ Department of Thoracic Surgery, Shanghai Pulmonary Hospital, Tongji University, Shanghai 200003, China; ${ }^{13}$ Shanghai Lung Tumor Clinical Medical Center, Shanghai Chest Hospital, Shanghai Jiao Tong University, Shanghai 200030, China; ${ }^{14}$ Department of Thoracic Surgery, Zhejiang Cancer Hospital, Hangzhou 310022, China; ${ }^{15}$ Department of Thoracic Surgery, Institute of Surgery Research, Daping Hospital, Third Military Medical University, Chongqing 400042, China; ${ }^{16}$ Department of Thoracic Surgery, Fujian Medical University Union Hospital, Fuzhou 350001, China; ${ }^{17}$ Department of Thoracic Surgery, Tangdu Hospital, The Fourth Military Medical University, Xi'an 710038 , China; ${ }^{18}$ Department of Thoracic Surgery, First Affiliated Hospital of Xinjiang Medical University, Urumqi 830054, China; ${ }^{19}$ Department of Thoracic Surgery, Nanjing Medical University Affiliated Cancer Hospital, Jiangsu Key Laboratory of Molecular and Translational Cancer Research, Cancer Institute of Jiangsu Province, Nanjing 210009, China; ${ }^{20}$ Department of Thoracic Surgery, Sun Yat-Sen University Cancer Center, Guangzhou 510060, China; ${ }^{21}$ Department of Thoracic Surgery, Beijing Chao-Yang Hospital, Beijing 100043, China; ${ }^{22}$ Department of Thoracic Surgery, Shanghai Zhongshan Hospital of Fudan University, Shanghai 200032, China; ${ }^{23}$ Department of Thoracic Surgery, China and Japan Friendship Hospital, Beijing 100029, China; ${ }^{24}$ Department of Lung Cancer Center, West China Hospital, Sichuan University, Chengdu 610041, China; ${ }^{25}$ Department of Thoracic Surgery, Tongji Hospital, Tongji Medical College, Huazhong University of Science and Technology, Wuhan 430030, China; ${ }^{26}$ Shandong Provincial Qianfoshan Hospital, Shandong University, Jinan 250014, China; ${ }^{27}$ Department of Thoracic Surgery, Fudan University Shanghai Cancer Center, Shanghai 200032, China; ${ }^{28}$ Department of Thoracic Surgery, Shanghai Chest Hospital, Jiao Tong University, Shanghai 200032, China; ${ }^{29}$ Department of Thoracic Surgery, Tianjin Chest Hospital, Tianjin 300051, China; ${ }^{30}$ Department of Thoracic Surgery, Second Hospital of Jilin University, Changchun 130041, China; ${ }^{31}$ Department of Esophageal Cancer, Tianjin Medical University Cancer Institute and Hospital, National Clinical Research Center for Cancer, Key Laboratory of Cancer Prevention and Therapy, Tianjin 300060, China; ${ }^{32}$ Department of Thoracic Surgery, Liaoning Cancer Hospital and Institute, Shenyang 110042, China; ${ }^{33}$ Department of Thoracic Surgery, Xuanwu Hospital of Capital Medical University, Beijing 100053, China; ${ }^{34}$ Department of Thoracic Surgery, Peking University Third Hospital, Beijing 100191, China; ${ }^{35}$ Department of Thoracic Surgery, The Second Hospital of Jilin University, Changchun 130041, China; ${ }^{36}$ Thoracic Surgery Department, Hospital 


\section{Page 2 of 12}

Liu et al. Consensus on managing bleeding during VATS lung surgery

Universitario Miguel Servet, IIS Aragón, Zaragoza, Spain; ${ }^{37}$ Department of Thoracic Surgery, Asturias University Central Hospital, Oviedo, Spain; ${ }^{38}$ Department of Thoracic Surgery, University of Health Sciences, Sureyyapasa Training and Research Hospital, Istanbul, Turkey; ${ }^{39}$ Division of Thoracic Surgery, Inselspital, University Hospital Bern, Bern, Switzerland; ${ }^{40}$ Department of Thoracic Surgery, Maggiore Teaching Hospital, Bologna, Italy; ${ }^{41}$ Department of Thoracic Surgery, St. James's University Hospital, Leeds, UK; ${ }^{42}$ Department of Thoracic Surgery, Coruña University Hospital and Minimally Invasive Thoracic Surgery Unit (UCTMI), Coruña, Spain; ${ }^{43}$ Department of Cardiothoracic Surgery, James Cook University Hospital, Middlesbrough, UK; ${ }^{44}$ Department of Thoracic Surgery, Chang Gung Memorial Hospital (Linkou), Taiwan, China; ${ }^{45}$ Department of Thoracic Surgery, Brigham and Women's Hospital, Boston, Massachusetts, USA; ${ }^{46}$ Department of Thoraco-abdominal Surgical Oncology. Herzen Moscow Cancer Research Center, Russia; ${ }^{47}$ Department of Cardiothoracic Surgery, University of Pittsburgh Medical Center, Pittsburgh, PA, USA; ${ }^{48}$ Honorary Consultant in Cardio-Thoracic Surgery, Gleneagles Hong Kong Hospital, Hong Kong, China; ${ }^{49}$ Department of Surgical Oncology, Nagasaki University Graduate School of Biomedical Sciences, Nagasaki, Japan; ${ }^{50}$ Department of General Thoracic Surgery, Dokkyo Medical University, Mibu, Tochigi, Japan; ${ }^{51}$ Department of Thoracic Surgery, Thoracoscopic Surgery Center, New Tokyo Hospital, Chiba, Japan; ${ }^{52}$ Agasthian Thoracic Surgery Pte Ltd. 3 Mount Elizabeth \#14-12 Mount Elizabeth Medical Centre, Singapore; ${ }^{53}$ Division of Thoracic Surgery, Department of Surgery, Cedars-Sinai Medical Center, Los Angeles, CA, USA; ${ }^{54}$ Moores Cancer Center, UC San Diego Health - La Jolla, Moores Cancer Center, La Jolla, USA; ${ }^{55}$ Division of Thoracic Surgery, Sun Yat-Sen Cancer Center, Taipei, Taiwan, China

Correspondence to: Lunxu Liu, MD, PhD. FRCS. Department of Thoracic Surgery, West China Hospital, Sichuan University, Chengdu 610041, China. Email: lunxu_liu@aliyun.com.

Abstract: Intraoperative bleeding is the most crucial safety concern of video-assisted thoracic surgery (VATS) for a major pulmonary resection. Despite the advances in surgical techniques and devices, intraoperative bleeding is still not rare and remains the most common and potentially fatal cause of conversion from VATS to open thoracotomy. Therefore, to guide the clinical practice of VATS lung surgery, we proposed the International Interest Group on Bleeding during VATS Lung Surgery with 65 experts from 10 countries in the field to develop this consensus document. The consensus was developed based on the literature reports and expert experience from different countries. The causes and incidence of intraoperative bleeding were summarised first. Seven situations of intraoperative bleeding were collected based on clinical practice, including the bleeding from massive vessel injuries, bronchial arteries, vessel stumps, and bronchial stumps, lung parenchyma, lymph nodes, incisions, and the chest wall. The technical consensus for the management of intraoperative bleeding was achieved on these seven surgical situations by six rounds of repeated revision. Following expert consensus statements were achieved: (I) Bleeding from major vascular injuries: direct compression with suction, retracted lung, or rolled gauze is useful for bleeding control. The size and location of the vascular laceration are evaluated to decide whether the bleeding can be stopped by direct compression or by ligation. If suturing is needed, the suction-compressing angiorrhaphy technique (SCAT) is recommended. Timely conversion to thoracotomy with direct compression is required if the operator lacks experience in thoracoscopic angiorrhaphy. (II) Bronchial artery bleeding: pre-emptive clipping of bronchial artery before bronchial dissection or lymph node dissection can reduce the incidence of bleeding. Bronchial artery bleeding can be stopped by compression with the suction tip, followed by the handling of the vascular stump with energy devices or clips. (III) Bleeding from large vessel stumps and bronchial stumps: bronchial stump bleeding mostly comes from accompanying bronchial artery, which can be clipped for hemostasis. Compression for hemostasis is usually effective for bleeding at the vascular stump. Otherwise, additional use of hemostatic materials, re-staple or a suture may be necessary. (IV) Bleeding from the lung parenchyma: coagulation hemostasis is the first choice. For wounds with visible air leakage or an insufficient hemostatic effect of coagulation, suturing may be necessary. (V) Bleeding during lymph node dissection: non-grasping en-bloc lymph node dissection is recommended for the nourishing vessels of the lymph node are addressed first with this technique. If bleeding occurs at the site of lymph node dissection, energy devices can be used for hemostasis, sometimes in combination with hemostatic materials. (VI) Bleeding from chest wall incisions: the chest wall incision(s) should always be made along the upper edge of the rib(s), with good hemostasis layer by layer. Recheck the incision for hemostasis before closing the chest is recommended. (VII) Internal chest wall bleeding: it can usually be managed with electrocoagulation. For 
diffuse capillary bleeding with the undefined bleeding site, compression of the wound with gauze may be helpful.

Keywords: Video-assisted thoracic surgery (VATS); hemorrhage; pulmonary resection; expert consensus

Submitted Nov 16, 2019. Accepted for publication Nov 25, 2019.

doi: 10.21037/atm.2019.11.142

View this article at: http://dx.doi.org/10.21037/atm.2019.11.142

\section{Introduction}

Surgeons expanded their indications for video-assisted thoracic surgery (VATS) to treat benign and malignant pulmonary diseases over the past two decades. For such maladies, many investigators demonstrated that VATS perioperative and long-term outcomes are at least noninferior to that of thoracotomy (1-16). Consensus recommendations from the National Comprehensive Cancer Network (NCCN, Plymouth Meeting, PA, USA) Clinical Practice Guidelines in Oncology, Non-small cell lung cancer (17) and the Chinese Guidelines on the Diagnosis and Treatment of Primary Lung Cancer (2015 version) (18) indicate that VATS and other minimally invasive approaches are preferred for lung cancer patients without surgical contraindications.

As an increasingly mature technology, VATS major pulmonary resections can be performed through threeportal, two-portal, or uniportal approaches $(19,20)$. In addition to routine lung cancer resections, it can also be applied for more challenging surgeries like bronchial sleeve lobectomy, bronchovascular double sleeve lobectomy (21-23), and even carinal resection (24). However, several factors may limit the application of VATS, including complete pleural symphysis, dense hilar adhesions (i.e., hilar fusion), vascular injuries/bleeding, and surgeon's difficulty in adapting to VATS procedure. In 2012, a global survey found half $(49.6 \%)$ of the responding 850 thoracic surgeons did not perform VATS lobectomy (25), mainly due to the lack of training opportunities; notably, 35 (4.1\%) of these thoracic surgeons refused to learn this technique because of safety concerns. Major bleeding is the most critical intraoperative safety concern of VATS subject to the surgeon's control. Because of the potential exposure limitations of VATS, the large lung blood vessel size with high flow, the anatomic density and variability, and the thin wall thickness, any vascular injury is dangerous and often difficult to control. Patients may quickly experience hemorrhagic shock and even death.
Bleeding, especially when caused by pulmonary vascular injuries, is a common and fundamental problem during lung surgery. Hemorrhage has been described as one of the most common and potentially fatal causes of conversion from VATS major pulmonary resection to open thoracotomy (26-29). The incidence of significant bleeding during VATS major pulmonary resection varies by regional differences in population characteristics, disease severities, and surgical center capacities. In 2016, Ghosh et al. retrospectively analyzed 8,563 cases of VATS anatomic lung resection based on a U.S. national database (Premier Perspective Database) and found that $9.2 \%$ of the patients required a blood transfusion due to intraoperative bleeding (30). These data were collected from more than 600 hospitals in the United States and reflected a broad-based national incidence sample of clinically significant bleeding during VATS. Other large medical centers reported the rates of massive bleeding from vascular injury during VATS major pulmonary resection ranging from $2.9 \%$ to $8.3 \%$ (29,31-36).

Among the options for massive bleeding during VATS lung surgery, urgent conversion to thoracotomy is used most commonly. In 2015, Decaluwe et al. studied 3,076 cases of VATS major pulmonary resection from six European centers (34). Among these cases, 88 (3\%) patients suffered from intraoperative bleeding due to vascular injury, and $70(79.5 \%)$ cases were converted to open surgery (34). A recent study of the Italian VATS lobectomy registry enrolled 1,679 cases from ten high-volume centers found that the bleeding-related conversion rate was $2.6 \%$ among all the patients (37). Massive bleeding from vascular injury prolongs hospital stay and increases hospitalization costs significantly (30). Uncommonly, emergent pneumonectomy is required to control bleeding, which may significantly increase the risk of perioperative mortality (34). International centers have reported timely and effective thoracoscopic control of massive bleeding with high VATS experience. Liu et al. recently reported the technical details of handling massive bleeding due to vascular injury during 
VATS pulmonary resection by vascular side-compression using an endoscopic suction, followed by repairing the injured vessels directly (36). Of the 17 vascular injuries, $88.7 \%$ were managed successfully without conversion (36). Besides major vascular lacerations, intraoperative VATS hemorrhage arises from bronchial arterial injury, partial pulmonary arterial or venous stump dehiscence, and lower capacity vessels dissected from the bronchial stump, lung parenchyma, lymph nodes, chest wall, and subcutaneous incisions.

Today, concerns about bleeding, especially that caused by central vascular injury, is the main factor limiting the expansion of VATS lung surgery. Unfortunately, there is no available expert consensus on this issue. Thus, we have organized the International Interest Group on Bleeding during VATS Lung Surgery to develop this expert consensus, with an attempt to optimize the management of this significant intraoperative complication.

\section{Methodology}

Dr. Liu L organized the 65 members of the International Interest Group. They were invited to participate in the working group voluntarily. All the members represented academic centers, large teaching hospitals, and community hospitals from Asia, Europe, and North America. The technical details of VATS lung surgery were analyzed and discussed at the initiation. Seven situations of intraoperative bleeding were listed to achieve the consensus according to clinical practice, including the bleeding from massive vessel injuries, bronchial arteries, vessel stumps, and bronchial stumps, lung parenchyma, lymph nodes, incisions, and the chest wall.

Relevant literature was searched using PubMed, Embase, and Web of Science to identify articles published in English since 1990. The search term was as followed: ((video-assisted thoracic surgery OR minimally invasive surgery OR VATS) AND (bleeding OR hemorrhage)) AND (pulmonary resection or lobectomy). A total of 628 publications addressed the problem of bleeding during VATS pulmonary resection. There were no randomized clinical trials (RCT). Most of the published relevant articles were surgical case series or technical reports due to the distinct field of the consensus. Levels of evidence and grades of recommendation have been applied from the Infectious Disease Society of America-United States Public Health Service Grading System (38). The statements and recommendations without grading were based on experts' consensus and accessible evidence. Quality of the evidence was divided into three levels (from I to III), while the grade of recommendation was classified into five-degree (from A to E). The focus of these articles was intraoperative bleeding due to vascular injury, while few articles discussed the other six topics. However, these six situations were also common clinical concerns after discussion. The technical strategies for the management of intraoperative bleeding were formed based on the published articles, in combination with the experts' clinical practice if no publications were available. The final consensus was achieved after six rounds of repeated revision by the members of the Group.

\section{Consensus}

\section{Management of bleeding from substantial vessel injuries during VATS lung surgery}

\section{Causes of major vascular injuries}

Major vascular injuries are the most challenging situation during VATS pulmonary resection. The injuries of the main pulmonary artery $(\mathrm{PA})$ or its branches are the most common causes of intraoperative bleeding. The other injuries reported originating from pulmonary veins, superior vena cava, azygos vein, inferior vena cava, abnormal vessels, and the thoracic aorta $(31,33,35,36)$. Since the PA has thin walls and large blood flow under low pressure (high capacitance), bleeding can be particularly fierce and uncontrollable and often requires conversion to thoracotomy (32,35,39-42). Intraoperative major vascular bleeding can be categorized as follows:

\section{PA injury}

The PA and its branches usually travel along with the bronchi. Tuberculosis, chronic infectious diseases, and other pathological factors cause chronic inflammation, calcification of hilar lymph nodes, and dense adhesions between the PA and bronchi, which are difficult to separate surgically and increase dissection-related vascular injury. Mei et al. (36) summarised 17 cases of major vascular injury bleeding during VATS major pulmonary resection. Most patients with pulmonary arterial injury had severe anthracofibrosis around bronchus or vessels as well as with dense hilar adhesions (36). PA injuries often occurred during sharp dissection with scissors. Also, accidental injuries were caused by energy devices (ultrasound scalpel or electrocautery hook), forceps, or suction instruments $(31,33,35)$. Furthermore, unclear plane and inadequate dissection of the PA branches and improper endo-stapler 
angulation adjustments may also lead to PA lacerations. In addition, unawareness of small PA branches that could not be detected by CT scan may sometimes lead to tearing and cause bleeding while dissecting the PA. In rare cases, mechanical failures of endo-staplers, slippage, or avulsions of vascular clips or loosening of sutures at the PA branch stumps also cause massive bleeding.

\section{Pulmonary vein injury}

The blood pressure of the pulmonary vein is low. Besides, the perivascular area of the pulmonary vein is usually loose and can be dissected easily. Therefore, the characteristics of the pulmonary vein make massive bleeding caused by venous injury uncommon. However, injuries can occur during dissection and can be challenging to achieve proximal and distal control because of fibrosis and relation to other central hilar structures.

\section{Injuries of superior vena cava and azygos venous system}

The injuries of the azygos vein, superior vena cava, and cephalobrachial (innominate) vein mainly occur during the dissection of lymph node stations $2 \mathrm{R}, 3 \mathrm{a}$, and $4 \mathrm{R}(35,36)$. The azygos vein may also be mistakenly injured when dissecting the pulmonary hilum.

Injuries of great thoracic arteries or anomalous arteries The injuries of great thoracic arteries or anomalous arteries are less common in lung surgeries. Yamashita $\mathrm{et} \mathrm{al}$. reported two cases of thoracic aorta injury, which occurred during intraoperative puncture biopsy for pulmonary lesions (35). Also, the thoracic aorta can be easily injured by the tip of energy devices during dissection of the pulmonary ligament. In addition, in patients with pulmonary sequestration, the arteries of the diseased lobe usually originate directly from systemic circulatory arteries such as the aorta, phrenic artery, intercostal artery, or celiac trunk. If the anomalous feeding artery was not diagnosed before surgery and/ or correctly recognized during the procedure, it might be mistaken as adhesions. The accidental injuries of the anomalous artery can result in massive bleeding.

\section{Management of massive bleeding during VATS lung surgery}

Bleeding-control after major vascular injury

Bleeding-controlling is the top priority in case of major vascular injury during a VATS pulmonary resection. In the event of bleeding, it is crucial to keep calm and to use compression as the first step. Compression is the most common and direct method for hemostasis, during which, suction, gauze balls, gauze, adjacent lung tissues, or surgical instruments may be used $(43,44)$.

During operative dissection, the suction often helps improve surgical field exposure by retraction, and smoke evacuates and blood aspiration. When bleeding occurs, the suction tip can be applied directly to compress the injured site to control bleeding (known as the suction-compressing maneuver), which also allows the effective removal of the accumulated blood and thus keep the surgical site clear $(36,45,46)$. In addition to the suction, gauze balls, gauzes, or wet gauze with a hemostatic agent can also be used for compression $(43,44)$. The gauze can be wrapped around the forceps or folded and clamped within a ring forceps to create a gauze bar for compression. Robotic surgeons use a rolled intracorporal sponge ("cigar") for the same purpose (47). In rare urgent cases, the adjacent lung tissue can be grasped and used for compression (33). If a vascular injury occurred when placing the endo-stapler, the bleeding could be temporarily controlled by reapplying compression with a portion (such as the front end) of the endo-stapler. Blind clamping for hemostasis should be avoided to prevent further damage to the vascular wall, which may make a simple repair impossible and exacerbate bleeding. A gauze ball or gauze, when applied for compression, will cover the target surgical field and make it difficult to repair the blood vessel under thoracoscopic vision.

In contrast, the suction device can be quickly placed and applied to control bleeding through the small operating holes on the chest wall. In addition to bleeding-control, the suction can also be used to clear up the blood and expose the injured blood vessel, leaving space for subsequent operation. It should be noted that the use of suction near the laceration without some compression can hasten to bleed (45). At this time, if suction or gauze cannot entirely stop bleeding, it must be converted to thoracotomy without delay.

\section{Management of vascular lacerations}

(I) Continuous compression and application of hemostatic materials: For bleeding from small lacerations of pulmonary arteries or veins during the blunt dissection, hemostasis may be achieved by sustained ( $\sim 5$ minutes) compression $(35,44)$. Advanced hemostatic materials such as collagen sponge or patch aid hemostasis and can temporarily close vascular injuries up to $6 \mathrm{~mm}$ to allow safer endoscopic control (33). In some cases, compression can be maintained for prolonged periods by occluding the area against the mediastinum or another fixed-site while dissection can proceed in another area of the anatomy to save time. In injuries of the PA when hemostasis has been achieved by 
simple compression, a small piece of the hemostatic patch can be applied over the tear to prevent the reopening and new bleeding.

(II) Ligation and/or clamping with titanium clips or locking clips: For bleeding near the branch of a blood vessel or that caused by the shedding of the ligating sutures, hemostasis could be achieved by clamping the lateral vascular wall or the vascular stump with a titanium clip or locking clip (45). Sometimes, the injured vessel could be transected with an endo-stapler.

(III) Suturing the injuries: Suturing the injured wall may be required for bleeding from vascular injuries that cannot be stopped by direct compression or clamping. Tools designed for minimally invasive cardiac surgery often work well.

Sometimes for a skilled operator, if the bleeding is not severe, the compression can be loosened to expose the bleeding site, and the suture should be completed as quickly as possible in the presence of bleeding.

Regardless of the volume of bleeding, suturing with adequate control of bleeding is a safe practice. The suctioncompressing angiorrhaphy technique (SCAT) $(36,46,48)$ can cope with most bleeding. The sidewall of the suction tip can be used for controlling bleeding. Furthermore, the suction can suck up the blood in the surgical field and help expose the injured site. After assessing the size and location of the vascular injury, different management strategies can be applied.

* Direct rotating-suture angiorrhaphy: this technique can be used if the vascular laceration is less than $5 \mathrm{~mm}$, and the suction-compressing maneuver can effectively control the bleeding. With the use of a 5-0 Polypropylene suture, one stitch is made at one side of the laceration after a slight rotation of the suction towards the laceration; then, a second stitch is made at the other side of the laceration after rotating the suction to the opposite side to complete the repair. After that, the knot can be tied.

* Clamping angiorrhaphy (edge-control): if the vascular laceration is greater than $5 \mathrm{~mm}$ but limited to $1 / 3$ of the vascular circumference, or if the angle of direct rotating-suture angiorrhaphy is weak, a pair of Allis forceps (or atraumatic clamp if available) can be used to gently clamp the edges of vascular wound to replace the suction for bleeding control. Then the vascular wound can be sutured and repaired under control of clamping.

* Blocking angiorrhaphy (inflow-occlusion): if the vascular laceration is large (exceeding $1 / 3$ of the vascular circumference) or if the Allis forceps hinders subsequent suturing after it clamps the blood vessel (usually seen in PA injury), the proximal end of the blood vessel can be further dissected and controlled with an atraumatic device such as an double-loop vascular sling (49), an endoscopic bulldog clamp $(46,48)$, or a variety of other open or endoscopic vascular clamps that may require creating another incision to introduce $(22,26)$. After the inflow bleeding is controlled, if necessary, the distal end of the blood vessel can also be similarly occluded. The clamping of the residual pulmonary vein is also useful for controlling the backflow of the bleeding from PA. Then, the vascular laceration can be sutured. If the laceration is large and close to the root of the PA, it may be necessary to open the pericardium to clamp the main PA inside the pericardium. Of all these techniques, inflow occlusion takes longer but offers the best opportunity to limit massive volume blood loss and may be safer if the surgeon has less access to endoscopic vascular suturing instruments or impaired exposure to the site of injury.

* Conversion to thoracotomy: the best policy for treating bleeding complications is to prevent them from happening. Conversion to thoracotomy is an essential measure for the management of bleeding due to vascular injury during VATS major pulmonary resection and should not be regarded as a surgical failure $(29,50,51)$. However, the surgical team must have a plan on how to deal with the bleeding before surgery. The operating room should be prepared with thoracotomy instruments. Conversion to thoracotomy, best done after compression of the bleeding site, should be performed for (i) large vascular laceration, massive hemorrhage, and inadequate endoscopic vision which prevent further thoracoscopic actions; (ii) extension of the primary vascular laceration during attempts of vascular repair; (iii) hemodynamic instability or severe hypovolemia; and (iv) insufficient surgeon or team experience or resources such as instrumentation to attempt VATS repair. Also, it should be noted that when handling the injury and bleeding of the pulmonary vein and its variant branches, the stump(s) may get retracted into the pericardium, which is particularly challenging to deal with; thus, timely conversion to a thoracotomy may be required. In general, it is beneficial to clean a bloodied 
thoracoscope and reinsert to optimize compression and bleeding control. Attempting thoracotomy during active bleeding should be discouraged as blood flow is often so significant that hemodynamic collapse will occur before the ribs can be spread. In rare cases of massive left chest bleeding with severe hypovolemia, clamping the descending aorta could help in maintaining the blood pressure.

\section{Recommendations}

For bleeding from major vascular injuries during VATS lung surgery, direct compression with a device previously designated as optimal for the mini-invasive approach is required. For surgeons who use a suction applied routinely for dissection, this tool may be the preferred bleeding control instrument, as described above. For other approaches, pushing retracted lung immediately on the injury or use of gauze applied precisely through the VATS access incision using a "sponge stick" is also popular. For robotic surgery, an intracorporal rolled gauze ("cigar") or assistant-based suction or lung retractor compression can be used. Many times, compression for 5 minutes or inserting an advanced hemostatic agent will be all that is needed. (Level II; Grade A).

After the bleeding is controlled, the operator must recall what kinds of vessel injury and think about what to do in the next step. The size and location of the vascular laceration are evaluated to decide whether the bleeding can be stopped by direct compression or by ligation with titanium clips. If suturing for hemostasis is needed, strategies including rotating-suture angiorrhaphy, clamping angiorrhaphy (Edge-control), and blocking angiorrhaphy (In-flow occlusion) can be applied to repair the vascular wound according to its size and location. If the operator lacks experience in thoracoscopic angiorrhaphy, timely conversion to thoracotomy is required after the bleeding is controlled by direct compression (Level II; Grade A).

\section{Bronchial artery bleeding}

Bronchial arteries usually originate from the descending aorta or aortic arch and enter the lungs along with the left and right main bronchi, nourishing all levels of the bronchial tree (52). Bleeding from the bronchial artery during a VATS major pulmonary resection is often caused by direct injury to the artery during bronchi and/or subcarinal lymph node dissections. The operator must have a solid knowledge of the anatomy of the bronchial arteries and take proactive measures to avoid bleeding. Particular attention should be paid to bronchial artery hyperplasia caused by bronchiectasis, pulmonary sequestration, and other chronic inflammatory conditions. The bronchial arteries that feed the subcarinal lymph nodes should be ligated carefully, sealed, or clipped during lymph node dissection. A bronchial artery can be injured during dissection of bronchi when chronic inflammation or calcification is present. When handling the left bronchial artery on the left side near its origin from the aorta, electrocoagulation must be avoided. Ligation or clips are preferred to prevent rebleeding. Although bronchial arteries have high pressure, bleeding is often controllable because these arteries are not significant and can be ligated with few risks. The bleeding can be controlled directly by compression with the head end of the suction and then stopped by electric coagulation, harmonic scalpel, or by clamping with titanium clips or polymer clips (31). Notably, a reoperation is sometimes required due to delayed bronchial artery bleeding near the lateral margin of the bronchial stump (53). Before incision closure, the entire operative field with attention to the bronchial artery should be carefully re-checked.

\section{Recommendations}

Pre-emptive clipping of the bronchial artery before bronchial dissection or lymph node dissection can reduce the incidence of bleeding. Bronchial artery bleeding can be stopped by compression with the suction tip, followed by the handling of the vascular stump with energy devices or clips. When using energy devices to stop bronchial artery bleeding, care should be taken to avoid injury to the membranous airway, carina, and esophagus (Level III; Grade B).

\section{Bleeding from large vessel stumps and bronchial stumps}

Bleeding from the stumps may occur after the endoscopic transection of bronchi, pulmonary arteries, and pulmonary veins with an endo-stapler. Bleeding from a bronchial stump is caused mostly by bleeding from a bronchial artery accompanying the bronchus, which can be stopped by clamping the proximal end of the bronchial artery with titanium clips or polymer clips. These clips can also be applied at the bronchial stump margin directly. For experienced operators, electrocoagulation at the resection margin of the bronchial stump is also acceptable for hemostasis. Notably, the resection margin of the bronchus can be managed by electrocoagulation only at the Spray 
mode. Any touch with the staples should be prevented to avoid staple damage or bronchial injury and leakage. In contrast, bleeding from vascular stumps is mainly due to a mismatch of the rather thin vessel walls on the one hand and a relatively high staple height on the other hand, so that the staples may not be able to close the resection margin satisfactorily (54). This occurs more commonly in superior veins. In some cases, vascular stumps can become inadvertently entrapped in stapler loads applied to the bronchi and be torn during the application or firing sequence. Compression with gauze for 1-3 minutes is often enough to stop such bleeding. Otherwise, the additional use of hemostatic material to cover the vascular stump can be used. If the bleeding cannot be effectively stopped by compression, polymer clips or titanium clips can be used to clamp the vessel stump. If the stump is too short for the application of clips, it can also be sutured with Polypropylene stitches to stop bleeding. An endoscopic vascular stapler can also be used to re-staple the stump if it is long enough.

\section{Recommendations}

Bronchial stump bleeding mostly comes from an accompanying bronchial artery. It is recommended that the proximal end of the bronchial artery is clamped for hemostasis. For bleeding at the vascular stump, compression for hemostasis is usually effective. If insufficient, the additional use of hemostatic material or, if still insufficient, clips, re-staple, or a suture may be necessary (Level III; Grade B).

\section{Bleeding from the lung parenchyma}

Pulmonary segmentectomy or lobectomy is usually performed by dividing the pulmonary tissues with endostaplers. Sometimes capillary bleeding can be seen at the resection margin, which may be explained by thin pulmonary tissue, the long-staple height of the staples (which makes it difficult to seal the pulmonary tissue completely), as well as the poor closure at the starting site of endo-staplers (55). In this case, electrocoagulation of the incision margin can be performed for hemostasis. For pulmonary laceration caused by grasping the lung tissue during surgery or bleeding from a pulmonary wound caused by blunt dissection of the lung parenchyma, electrocoagulation can also be applied, combined with coverage of the wound with hemostatic materials. When using cautery to stop parenchymal bleeding, it is better to use on the highest setting of coagulation (most devices this is 100) and arc the energy onto the surface of the bleeding lung in a similar way that one might use the argon beam electrocoagulation. If there is a visible air leak or if the hemostatic effect of electrocoagulation is unreliable, suturing with polydioxanone or Polypropylene stitches may be performed.

\section{Recommendations}

Coagulation hemostasis using a variety of devices is the first choice for pulmonary parenchyma bleeding. For wounds with visible air leakage or an insufficient hemostatic effect of coagulation, suturing may be necessary, if required, in combination with hemostatic material to cover the wound (Level III; Grade B).

\section{Bleeding during lymph node dissection}

Bleeding during lymph node dissection includes bleeding due to lymph node fragmentation, oozing from the wound surface, and bleeding from injured adjacent vessels. Nongrasping en bloc mediastinal lymph node dissection (56), therefore, is recommended, during which the small blood vessels entering and leaving the lymph nodes are pretreated at the periphery of the lymph node stations, and then the lymph nodes and their adjacent connective tissues are removed en-bloc to avoid fragmentation and bleeding caused by grasping the lymph nodes inside the stations. If fragmentation occurs during dissection and the bleeding cannot be stopped through the application of pressure or an energy device, it is necessary to remove the remaining lymph nodes to achieve satisfactory hemostasis. Clips may reduce the rate of lymphatic leak, especially in regions near the thoracic duct. When an energy device is used to stop bleeding, any damage to adjacent structures, including the trachea, bronchus, and recurrent laryngeal nerve, should be avoided. If necessary, hemostatic materials can be used to assist hemostasis. Also, lymph node dissection may cause adjacent vascular injury/bleeding, which usually results from an injury of a bronchial artery during the dissection of subcarinal lymph nodes (45). The operator should know well about the anatomy of the bronchial arteries and clip them in advance. Occasionally, a variant branch of the pulmonary vein may directly enter the left atrium at the subcarinal area (57), which should be carefully handled during dissection of subcarinal lymph nodes. Once the vein stump retracts into the pericardium, thoracoscopic hemostasis will become difficult, and conversion to 
thoracotomy and pericardiotomy may be required. During the dissection of lymph node stations $2 \mathrm{R}$ and $4 \mathrm{R}$, there are small venous branches draining into the superior vena cava. These small veins should be carefully identified and adequately handled with clips, energy devices like ultrasound scalpel, or electrocautery to avoid bleeding. If they were mistakenly transected and cause bleeding, the suction-compressing maneuver could be applied for bleeding control. If the stumps are long enough, Harmonic scalpel or vascular clips may be applied; if there is no visible stump, the bleeding can be stopped by suturing.

\section{Recommendations}

Non-grasping en-bloc lymph node dissection is recommended since, with this technique, the nourishing vessels of the whole lymph node station are addressed first, which may help prevent bleeding from the lymph nodes during further dissection. If bleeding occurs at the site of lymph node dissection, energy devices can be used for hemostasis, and the oozing site can be covered with hemostatic materials if necessary. Bleeding from peripheral small blood vessels can be managed with energy instruments or vascular clips. Suturing for hemostasis may be needed in a small number of cases (Level III; Grade B).

\section{Bleeding from chest wall incisions}

Bleeding from a chest wall incision occasionally occurs (58). In general, the main problem is not the bleeding itself, but rather that the blood oozing from the incision flows into the thoracic cavity and/or spills and blurs the lens of the thoracoscope, subsequently affecting the vision and exposure of the surgical field. The main sources of bleeding from the incisions include small blood vessels from dissected muscle tissue or the intercostal vessels. When making the incisions, the soft tissues of the chest wall should be carefully divided layer by layer, along with adequate hemostasis, usually using an electrocautery device. The division of the intercostal muscles should always be performed at the upper edge of the ribs to avoid any injury to the intercostal vessels. If electrocoagulation fails to stop bleeding from chest wall muscle-feeding blood vessels, ligation or suturing for hemostasis may be applied. For small bleeding from the incision, the placement of a wound protector is usually sufficient for achieving hemostasis through slight compression (59). Careful examination of any bleeding from the incision should be done upon the completion of surgery; in particular, the incision shall be re- checked under the endoscopic vision to avoid re-operation for hemostasis due to incisional bleeding.

\section{Recommendations}

The chest wall incision(s) should always be made along the upper edge of the rib(s), with good hemostasis layer by layer. Any bleeding from an incision shall be carefully checked and managed appropriately before the occasional insertion of a wound protector. Furthermore, the incision site should always be rechecked for hemostasis upon completion of the procedure (Level III; Grade B).

\section{Internal chest wall bleeding}

Chest wall bleeding is seen in patients with thoracic adhesions or those undergoing extrapleural dissection or formal parietal pleurectomy. When handling the adhesion bundles, special attention should be paid to vascular bleeding within the adhesions, and the adhesion area should be adequately cauterized or clipped when dissecting the adhesions. For patients with extensive thoracic adhesions, it is necessary to find the right plane in the process of freeing the adhesions, free between the visceral layer and the parietal pleura, and the bleeding should be stopped adequately at each step to reduce bleeding from chest wall wounds. Because of the complex anatomy of the thoracic apex, dissection should be performed only with good exposure, to avoid any inadvertent injury to the large blood vessels in this region (60). For bleeding from the chest wall, the preferred hemostasis method is electrocoagulation; for more diffuse capillary bleeding or if the bleeding site is not identified, compressing the wound with gauze or a hemostatic agent may help to stop bleeding, followed by the handling of the apparent bleeding site. Extensive areas of chest wall oozing prevent good visualization and create massive aggregate blood loss. The use of the saline-coupled bipolar electrocautery or suction-coagulator devices may be beneficial for control bleeding from adhesions and a wide area of extrapleural dissection (45).

\section{Recommendations}

Bleeding from the inside of the chest wall can usually be managed with electrocoagulation. The optimal plane for dissection should be identified when dissecting any pleuropulmonary adhesions, and adequate hemostasis should be achieved at each step. For diffuse capillary bleeding with the undefined bleeding site, compression of the wound with gauze may help to stop bleeding (Level 
III; Grade B).

\section{Acknowledgments}

We thank Ms. Xueqin Tang, who helped us organizing the Interest Group. We thank Dr. Nan Chen and Dr. Zihuai Wang, who helped us screening the literature.

\section{Footnote}

Conflicts of Interest: The authors have no conflicts of interest to declare.

Ethical Statement: The authors are accountable for all aspects of the work in ensuring that questions related to the accuracy or integrity of any part of the work are appropriately investigated and resolved.

\section{References}

1. Onaitis MW, Petersen RP, Balderson SS, et al.

Thoracoscopic lobectomy is a safe and versatile procedure: experience with 500 consecutive patients. Ann Surg 2006;244:420-5.

2. McKenna RJ, Jr., Houck W, Fuller CB. Video-assisted thoracic surgery lobectomy: experience with 1,100 cases. Ann Thorac Surg 2006;81:421-5; discussion 425-6.

3. $\mathrm{Pu} \mathrm{Q}, \mathrm{Ma} \mathrm{L}, \mathrm{Che} \mathrm{GW}$, et al. Safety and technical feasibility of single-direction VATS lobectomy: a review of 1040 cases. Sichuan Da Xue Xue Bao Yi Xue Ban 2013;44:109-13.

4. Liu C, Pu Q, Ma L, et al. Video-assisted thoracic surgery for pulmonary sequestration compared with posterolateral thoracotomy. J Thorac Cardiovasc Surg 2013;146:557-61.

5. Forti Parri SN, Bonfanti B, Cancellieri A, et al. Molecular analysis driven video-assisted thoracic surgery resections in bilateral synchronous lung cancers: from the test tube to the operatory room. Ann Transl Med 2017;5:397.

6. Yang CJ, Kumar A, Klapper JA, et al. A National Analysis of Long-term Survival Following Thoracoscopic Versus Open Lobectomy for Stage I Non-small-cell Lung Cancer. Ann Surg 2019;269:163-71.

7. Yang CF, Sun Z, Speicher PJ, et al. Use and Outcomes of Minimally Invasive Lobectomy for Stage I Non-Small Cell Lung Cancer in the National Cancer Data Base. Ann Thorac Surg 2016;101:1037-42.

8. Pages PB, Delpy JP, Orsini B, et al. Propensity Score Analysis Comparing Videothoracoscopic Lobectomy With
Thoracotomy: A French Nationwide Study. Ann Thorac Surg 2016;101:1370-8.

9. Nwogu CE, D'Cunha J, Pang H, et al. VATS lobectomy has better perioperative outcomes than open lobectomy: CALGB 31001, an ancillary analysis of CALGB 140202 (Alliance). Ann Thorac Surg 2015;99:399-405.

10. Murakawa T, Ichinose J, Hino H, et al. Long-term outcomes of open and video-assisted thoracoscopic lung lobectomy for the treatment of early stage non-small cell lung cancer are similar: a propensity-matched study. World J Surg 2015;39:1084-91.

11. Cao C, Zhu ZH, Yan TD, et al. Video-assisted thoracic surgery versus open thoracotomy for non-small-cell lung cancer: a propensity score analysis based on a multi-institutional registry. Eur J Cardiothorac Surg 2013;44:849-54.

12. Paul S, Sedrakyan A, Chiu YL, et al. Outcomes after lobectomy using thoracoscopy vs thoracotomy: a comparative effectiveness analysis utilizing the Nationwide Inpatient Sample database. Eur J Cardiothorac Surg 2013;43:813-7.

13. Yan TD, Black D, Bannon PG, et al. Systematic review and meta-analysis of randomized and nonrandomized trials on safety and efficacy of video-assisted thoracic surgery lobectomy for early-stage non-small-cell lung cancer. J Clin Oncol 2009;27:2553-62.

14. Paul S, Isaacs AJ, Treasure T, et al. Long term survival with thoracoscopic versus open lobectomy: propensity matched comparative analysis using SEER-Medicare database. BMJ 2014;349:g5575.

15. Mei J, Guo C, Xia L, et al. Long-term survival outcomes of video-assisted thoracic surgery lobectomy for stage I-II non-small cell lung cancer are more favorable than thoracotomy: a propensity score-matched analysis from a high-volume center in China. Translational Lung Cancer Research 2019;8:155-66.

16. Bendixen M, Jorgensen OD, Kronborg C, et al. Postoperative pain and quality of life after lobectomy via video-assisted thoracoscopic surgery or anterolateral thoracotomy for early stage lung cancer: a randomised controlled trial. Lancet Oncol 2016;17:836-44.

17. NCCN Clinical Practice Guidelines in Oncology (NCCN Guidelines $\left.{ }^{\circledR}\right)$ Non-Small Cell Lung Cancer Version 2. 2018. National Comprehensive Cancer Network. 2017. Available online: https://www.nccn.org/professionals/ physician_gls/pdf/nscl.pdf.

18. Zhi XY, Yu JM, Shi YK. Chinese Guidelines on the Diagnosis and Treatment of Primary Lung Cancer (2015 
Version). Cancer 2015;121:Suppl 17:3165-81.

19. Liu L, Che G, Pu Q, et al. A new concept of endoscopic lung cancer resection: Single-direction thoracoscopic lobectomy. Surg Oncol 2010;19:e71-7.

20. Gonzalez-Rivas D, Fieira E, Mendez L, et al. Single-port video-assisted thoracoscopic anatomic segmentectomy and right upper lobectomy. Eur J Cardiothorac Surg 2012;42:e169-71.

21. Mahtabifard A, Fuller CB, McKenna RJ, Jr. Video-assisted thoracic surgery sleeve lobectomy: a case series. Ann Thorac Surg 2008;85:S729-32.

22. Mei J, Pu Q, Liao H, et al. Initial experience of videoassisted thoracic surgery left upper sleeve lobectomy for lung cancer: Case report and literature review. Thorac Cancer 2012;3:348-52.

23. Liu L, Mei J, Pu Q, et al. Thoracoscopic bronchovascular double sleeve lobectomy for non-small-cell lung cancer. Eur J Cardiothorac Surg 2014;46:493-5.

24. Lyscov A, Obukhova T, Ryabova V, et al. Double-sleeve and carinal resections using the uniportal VATS technique: a single centre experience. J Thorac Dis 2016;8:S235-41.

25. Cao C, Tian DH, Wolak K, et al. Cross-sectional survey on lobectomy approach (X-SOLA). Chest 2014;146:292-8.

26. Watanabe A, Koyanagi T, Nakashima S, et al. How to clamp the main pulmonary artery during video-assisted thoracoscopic surgery lobectomy. Eur J Cardiothorac Surg 2007;31:129-31.

27. Shaw JP, Dembitzer FR, Wisnivesky JP, et al. Videoassisted thoracoscopic lobectomy: state of the art and future directions. Ann Thorac Surg 2008;85:S705-9.

28. Congregado M, Merchan RJ, Gallardo G, et al. Videoassisted thoracic surgery (VATS) lobectomy: 13 years' experience. Surg Endosc 2008;22:1852-7.

29. Sawada S, Komori E, Yamashita M. Evaluation of videoassisted thoracoscopic surgery lobectomy requiring emergency conversion to thoracotomy. Eur J Cardiothorac Surg 2009;36:487-90.

30. Ghosh SK, Roy S, Daskiran M, et al. The clinical and economic burden of significant bleeding during lung resection surgery: A retrospective matched cohort analysis of real-world data. J Med Econ 2016;19:1081-6.

31. Igai H, Kamiyoshihara M, Ibe T, et al. Troubleshooting for bleeding in thoracoscopic anatomic pulmonary resection. Asian Cardiovasc Thorac Ann 2017;25:35-40.

32. Vallance A, Tcherveniakov P, Bogdan C, et al. The evolution of intraoperative conversion in video assisted thoracoscopic lobectomy. Ann R Coll Surg Engl 2017;99:129-33.
33. Miyazaki T, Yamasaki N, Tsuchiya T, et al. Management of unexpected intraoperative bleeding during thoracoscopic pulmonary resection: a single institutional experience. Surg Today 2016;46:901-7.

34. Decaluwe H, Petersen RH, Hansen H, et al. Major intraoperative complications during video-assisted thoracoscopic anatomical lung resections: an intention-totreat analysis. Eur J Cardiothorac Surg 2015;48:588-98; discussion 99.

35. Yamashita S, Tokuishi K, Moroga T, et al. Totally thoracoscopic surgery and troubleshooting for bleeding in non-small cell lung cancer. Ann Thorac Surg 2013;95:994-9.

36. Mei J, Pu Q, Liao H, et al. A novel method for troubleshooting vascular injury during anatomic thoracoscopic pulmonary resection without conversion to thoracotomy. Surg Endosc 2013;27:530-7.

37. Bertolaccini L, Davoli F, Pardolesi A, et al. Conversion due to vascular injury during video-assisted thoracic surgery lobectomy: A multicentre retrospective analysis from the Italian video-assisted thoracic surgery group registry. Eur J Surg Oncol 2019;45:857-62.

38. Gross PA, Barrett TL, Dellinger EP, et al. Purpose of quality standards for infectious diseases. Infectious Diseases Society of America. Clin Infect Dis 1994;18:421.

39. Puri V, Patel A, Majumder K, et al. Intraoperative conversion from video-assisted thoracoscopic surgery lobectomy to open thoracotomy: a study of causes and implications. J Thorac Cardiovasc Surg 2015;149:55-61, 2.e1.

40. Byun CS, Lee S, Kim DJ, et al. Analysis of Unexpected Conversion to Thoracotomy During Thoracoscopic Lobectomy in Lung Cancer. Ann Thorac Surg 2015;100:968-73.

41. Fournel L, Zaimi R, Grigoroiu M, et al. Totally thoracoscopic major pulmonary resections: an analysis of perioperative complications. Ann Thorac Surg 2014;97:419-24.

42. Samson P, Guitron J, Reed MF, et al. Predictors of conversion to thoracotomy for video-assisted thoracoscopic lobectomy: a retrospective analysis and the influence of computed tomography-based calcification assessment. J Thorac Cardiovasc Surg 2013;145:1512-8.

43. Demmy TL, James TA, Swanson SJ, et al. Troubleshooting Video-Assisted Thoracic Surgery Lobectomy. the Annals of Thoracic Surgery 2005;79:1744-52.

44. Berry MF. Pulmonary Artery Bleeding During VideoAssisted Thoracoscopic Surgery: Intraoperative Bleeding and Control. Thoracic Surgery Clinics 2015;25:239-47. 
45. Gonzalez-Rivas D, Stupnik T, Fernandez R, et al. Intraoperative bleeding control by uniportal video-assisted thoracoscopic surgerydagger. Eur J Cardiothorac Surg 2016;49 Suppl 1:117-24.

46. Xiao ZL, Mei JD, Pu Q, et al. Technical strategy for dealing with bleeding during thoracoscopic lung surgery. Ann Cardiothorac Surg 2014;3:213-5.

47. Louie BE. Catastrophes and complicated intraoperative events during robotic lung resection. J Vis Surg 2017;3:52.

48. Guo C, Mei J, Ma L, et al. Handling vascular bleeding without conversion during video-assisted thoracoscopic surgery major pulmonary resection. Ann Transl Med 2018;6:363.

49. Kohno T. Management of complications in thoracoscopic surgery. J Thorac Dis 2018;10:S1620-3.

50. Park JS, Kim HK, Choi YS, et al. Unplanned conversion to thoracotomy during video-assisted thoracic surgery lobectomy does not compromise the surgical outcome. World J Surg 2011;35:590-5.

51. Jones RO, Casali G, Walker WS. Does failed video-assisted lobectomy for lung cancer prejudice immediate and long-term outcomes? Ann Thorac Surg 2008;86:235-9.

52. Walker CM, Rosado-de-Christenson ML, MartinezJimenez S, et al. Bronchial arteries: anatomy, function, hypertrophy, and anomalies. Radiographics 2015;35:32-49.
53. McLean RH, Parandian BB, Nam MH. Pericardial tamponade: an unusual complication of lobectomy for lung cancer. Ann Thorac Surg 1999;67:545-6.

54. Craig SR, Walker WS. Potential complications of vascular stapling in thoracoscopic pulmonary resection. Ann Thorac Surg 1995;59:736-7; discussion 737-8.

55. Cerfolio RJ, Bryant AS, Skylizard L, et al. Initial consecutive experience of completely portal robotic pulmonary resection with 4 arms. J Thorac Cardiovasc Surg 2011;142:740-6.

56. Liu C, Pu Q, Guo C, et al. Non-grasping en bloc mediastinal lymph node dissection for video-assisted thoracoscopic lung cancer surgery. BMC Surg 2015;15:38.

57. Prasanna L C, R. P, D'Souza AS, et al. Variations in the pulmonary venous ostium in the left atrium and its clinical importance. J Clin Diagn Res 2014;8:10-1.

58. Yano M, Yokoi K, Numanami H, et al. Complications of bronchial stapling in thoracic surgery. World J Surg 2014;38:341-6.

59. Tanaka Y, Tane S, Hokka D, et al. The Use of Oxidized Regenerated Cellulose for Video-Assisted Thoracic Surgery. Ann Thorac Surg 2016;101:786-8.

60. Wang J, Liu TL, Chen HY. Management of pleural adhesion in the video-assisted thoracoscopic surgery. Zhonghua Wai Ke Za Zhi 1994;32:592-4.
Cite this article as: Liu L, Mei J, He J, Demmy TL, Gao S, Li S, He J, Liu Y, Huang Y, Xu S, Hu J, Chen L, Zhu Y, Luo Q, Mao W, Tan Q, Chen C, Li X, Zhang Z, Jiang G, Xu L, Zhang L, Fu J, Li H, Wang Q, Liu D, Tan L, Zhou Q, Fu X, Jiang Z, Chen H, Fang W, Zhang X, Li Y, Tong T, Yu Z, Liu Y, Zhi X, Yan T, Zhang X, Pu Q, Che G, Lin Y, Ma L, Embun R, Aragón J, Evman S, Kocher GJ, Bertolaccini L, Brunelli A, Gonzalez-Rivas D, Dunning J, Liu HP, Swanson SJ, Borisovich RA, Sarkaria IS, Sihoe ADL, Nagayasu T, Miyazaki T, Chida M, Kohno T, Thirugnanam A, Soukiasian HJ, Onaitis MW, Liu CC; on behalf of International Interest Group on Bleeding during VATS Lung Surgery. International expert consensus on the management of bleeding during VATS lung surgery. Ann Transl Med 2019;7(23):712. doi: 10.21037/atm.2019.11.142 